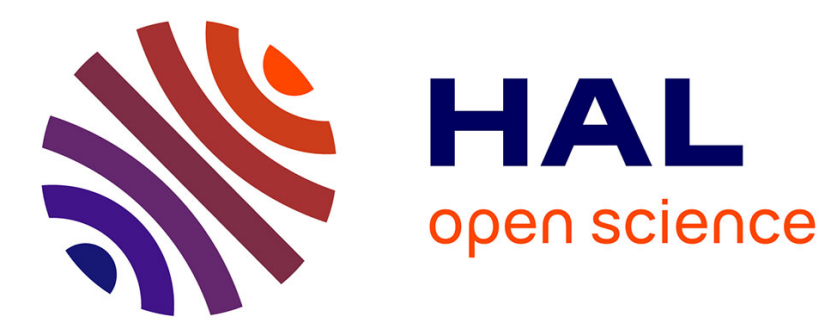

\title{
Propriétés d'un spectomètre $\beta$ à double focalisation, à secteurs Première Partie
}

S. Caeymaex, R. Boving, A. Jones, A. Martegani

\section{To cite this version:}

S. Caeymaex, R. Boving, A. Jones, A. Martegani. Propriétés d'un spectomètre $\beta$ à double focalisation, à secteurs Première Partie. Revue de Physique Appliquée, 1969, 4 (1), pp.1-5. 10.1051/rphysap:01969004010100 . jpa-00242882

\section{HAL Id: jpa-00242882 https://hal.science/jpa-00242882}

Submitted on 1 Jan 1969

HAL is a multi-disciplinary open access archive for the deposit and dissemination of scientific research documents, whether they are published or not. The documents may come from teaching and research institutions in France or abroad, or from public or private research centers.
L'archive ouverte pluridisciplinaire HAL, est destinée au dépôt et à la diffusion de documents scientifiques de niveau recherche, publiés ou non, émanant des établissements d'enseignement et de recherche français ou étrangers, des laboratoires publics ou privés. 


\title{
REVUE DE PHYSIQUE APPLIQUÉE
}

Supplément au "Journal de Physique "

\section{PROPRIÉTÉS D'UN SPEGTROMÈTRE $\beta$ A DOUBLE FOGALISATION, A SECTEURS Première Partie}

\author{
Par S. CAEYMAEX $\left({ }^{1}\right)$, R. BOVING, A. JONES et A. MARTEGANI,
} Centre de Physique Nucléaire, Université de Louvain.

(Reçu le 19 janviev 1968.)

Résumé. - On a calculé la résolution d'un spectromètre à deux secteurs, l'un à champ non uniforme, l'autre à champ uniforme, en fonction des angles de ceux-ci. La construction de ce spectromètre a été réalisée en vue de son utilisation, la source étant placée dans le faisceau d'un accélérateur.

Abstract. - The performance of a double-focusing sector-type $\beta$-spectrometer has been calculated. The spectrometer is composed of two sectors, one with homogeneous field and another with inhomogeneous field. The purpose is to use this apparatus on line with an accelerator.

PREMIERE PARTIE

Calculs du prisme

1. Introduction. - Le type de prisme considéré [1], [2], est formé d'un secteur à champ non uniforme suivi d'un secteur à champ uniforme (fig. 1). Dans le plan

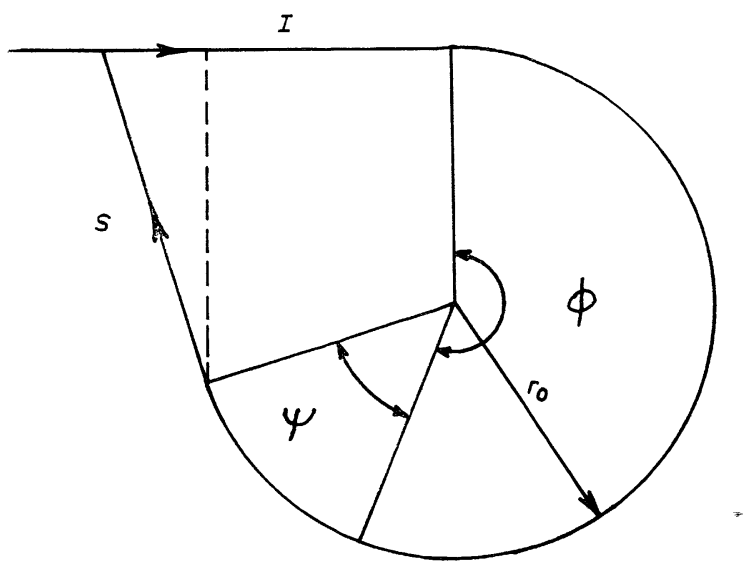

FIG. 1. - I, faisceau incident; S, faisceau sortant; $\Phi$, secteur à champ non uniforme $\left(200^{\circ}\right) ; \Psi^{*}$, secteur à champ uniforme $\left(40^{\circ}\right)$.

de symétrie de la partie non uniforme, le champ est normal à ce plan et a pour valeur :

$$
B=B_{0}\left[1+a \frac{x_{1}}{r_{0}}+b\left(\frac{x_{1}}{r_{0}}\right)^{2}+\ldots\right]
$$

(1) Actuellement : Division I.S.R. ; C.E.R.N., Genève (Suisse). où $r_{0}$ représente le rayon de la trajectoire centrale $\left(B=B_{0}\right)$ et $x_{1}$ est la coordonnée prise sur un axe ayant son origine sur la trajectoire $r_{0}$ et dirigé suivant le rayon $r_{0}$ vers le centre de cette circonférence ( $f i g .2$ ).

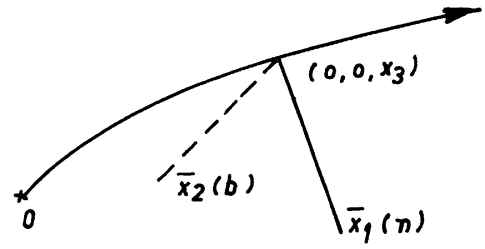

FIG. 2.

Pour un prisme quelconque, l'hamiltonien développé jusqu'au troisième ordre est :

$$
\begin{aligned}
H\left(x_{1}, x_{2}, x_{3},\right. & \left.q_{1}, q_{2}\right)=x_{1}\left[\frac{p}{r_{0}}+e B_{2}\left(0,0, x_{3}\right)\right] \\
& +\frac{x_{1}^{2}}{2}\left[e\left(\frac{\partial B_{2}}{\partial x_{1}}\right)_{0,0, x_{3}}-e \frac{B_{2}\left(0,0, x_{3}\right)}{r_{0}}\right] \\
& -\frac{x_{2}^{2}}{2} e\left(\frac{\partial B_{2}}{\partial x_{1}}\right)_{0,0, x_{3}}+\frac{q_{1}^{2}}{2 p}+\frac{q_{2}^{2}}{2 p} \\
& +\frac{x_{1}^{3}}{6}\left[e\left(\frac{\partial^{2} B_{2}}{\partial x_{1}^{2}}\right)_{0,0, x_{3}}-\frac{2 e}{r_{0}}\left(\frac{\partial B_{2}}{\partial x_{1}}\right)_{0,0, x_{3}}\right] \\
& +\frac{x_{1} x_{2}^{2}}{2}\left[\frac{e}{2}\left(\frac{\partial^{2} B_{2}}{\partial x_{2}^{2}}\right)_{0,0, x_{3}}+\frac{e}{2 r_{0}}\left(\frac{\partial B_{2}}{\partial x_{1}}\right)_{0,0, x_{3}}-\frac{e}{2}\left(\frac{\partial^{2} B_{2}}{\partial x_{1}^{2}}\right)_{0,0, x_{3}}\right] \\
& -\frac{x_{1}}{2 p r_{0}}\left(q_{1}^{2}+q_{2}^{2}\right) .
\end{aligned}
$$


2. Mouvement dans l'entrefer. - Cas général pour $B=B_{0}\left(1+a \frac{x_{1}}{r_{0}}\right) \quad[3]$, [4].

On recherche d'abord la solution monochromatique au premier ordre. Ensuite, par une méthode de perturbations, les aberrations géométriques et chromatiques $(\mathrm{d} p)$ ont été calculées.

a) Solution monochromatique. - Pour les coordonnées $x_{1}$ et $x_{2}$ et les impulsions conjuguées $q_{1}$ et $q_{2}$, ces solutions sont :

$$
\begin{array}{ll}
x_{1}=x_{10} u+q_{10} v ; & q_{1}=p_{0} x_{10} u^{\prime}+p_{0} q_{10} v^{\prime} \\
x_{2}=x_{20} w+q_{20} t ; & q_{2}=p_{0} x_{20} w^{\prime}+p_{0} q_{20} t^{\prime}
\end{array}
$$

avec :

$$
q_{10}=p_{0} x_{10}^{\prime} ; \quad q_{20}=p_{0} x_{20}^{\prime} ; \quad x_{10} ; \quad x_{20}
$$

les valeurs des impulsions conjuguées et des coordonnées à l'entrée du spectromètre $\left(x_{3}=0\right)$ et : $u=\cos \frac{(1-a)^{1 / 2} x_{3}}{r_{0}} ; \quad v=r_{0} \frac{\sin \left[(1-a)^{1 / 2} x_{3} / r_{0}\right]}{p_{0}(1-a)^{1 / 2}}$

$$
w=\cos \frac{\sqrt{a} \cdot x_{3}}{r_{0}} ; \quad t=r_{0} \frac{\sin \frac{\sqrt{a} \cdot x_{3}}{r_{0}}}{p_{0} \sqrt{a}} .
$$

b) Pour tenir compte des aberrations, on utilise une méthode de variation de constantes. La solution formelle est du type :

$$
\begin{array}{ll}
x_{1}=X_{10}\left(x_{3}\right) \cdot u & +Q_{10}\left(x_{3}\right) \cdot v \\
q_{1}=p_{0} X_{10}\left(x_{3}\right) \cdot u^{\prime} & +p_{0} Q_{10}\left(x_{3}\right) \cdot v^{\prime} \\
x_{2}=X_{20}\left(x_{3}\right) \cdot w & +Q_{20}\left(x_{3}\right) \cdot t \\
q_{2}=p_{0} X_{20}\left(x_{3}\right) \cdot w^{\prime}+p_{0} Q_{20}\left(x_{3}\right) \cdot t^{\prime} .
\end{array}
$$

Cette transformation étant canonique, l'évolution des nouvelles variables est définie par un nouvel hamiltonien $K$, tel que :

$$
\begin{aligned}
K\left(X_{10}, X_{20}, Q_{10}, Q_{20}, x_{3}, \mathrm{~d} p\right) & =H\left(x_{1}, x_{2}, x_{3}, q_{1}, q_{2}, \mathrm{~d} p\right)+\frac{\mathrm{d} F}{\mathrm{~d} x_{3}}
\end{aligned}
$$

où $F$ est la fonction génératrice de la transformation (3). Les coefficients satisfont dès lors aux équations :

$$
\begin{aligned}
\frac{\mathrm{d} X_{10}}{\mathrm{~d} x_{3}} & =\frac{\partial K}{\partial Q_{10}} ; & \frac{\mathrm{d} Q_{10}}{\mathrm{~d} x_{3}} & =-\frac{\partial K}{\partial X_{10}} \\
\frac{\mathrm{d} X_{20}}{\mathrm{~d} x_{3}} & =\frac{\partial K}{\partial Q_{20}} ; & \frac{\mathrm{d} Q_{20}}{\mathrm{~d} x_{3}} & =-\frac{\partial K}{\partial X_{20}} .
\end{aligned}
$$

L'hamiltonien $H$ est alors décomposé en $H_{1}+H_{2}$, où $H_{1}$ se rapporte au système monochromatique du premier ordre et $H_{2}$ est formé des nouveaux termes. Dans la recherche de la solution de (4), $H_{1}+\frac{\mathrm{d} F}{\mathrm{~d} x_{3}}$ qui n'est fonction que de $x_{3}$ ne doit pas être retenu. Le nouvel hamiltonien étant :

$K=\left(X_{10} u+Q_{10} v\right) \frac{\mathrm{d} p}{r_{0}}+$ termes d'ordres supérieurs on obtient au premier ordre :

$$
\begin{array}{ll}
X_{10}^{\prime}=\frac{v \mathrm{~d} p}{r_{0}} ; & X_{20}^{\prime}=0 \\
Q_{10}^{\prime}=-\frac{u \mathrm{~d} p}{r_{0}} ; & Q_{20}^{\prime}=0 .
\end{array}
$$

La solution complète, au premier ordre, est alors : $x_{1}=\left[x_{10}+\mathrm{d} p \int_{0}^{x_{3}} v \frac{\mathrm{d} x_{3}}{r_{0}}\right] u+\left[p x_{10}^{\prime}-\mathrm{d} p \int_{0}^{x_{3}} u \frac{\mathrm{d} x_{3}}{r_{0}}\right] v$ $x_{2}=x_{20} w+p_{0} x_{20}^{\prime} t$.

Les matrices correspondantes $M_{1}$ et $M_{2}$ :

$$
\left|\begin{array}{c}
x_{1} \\
x_{1}^{\prime} \\
\frac{\mathrm{d} p}{p_{0}}
\end{array}\right|=M_{1}\left|\begin{array}{c}
x_{10} \\
x_{10}^{\prime} \\
\frac{\mathrm{d} p}{p_{0}}
\end{array}\right| ; \quad\left|\begin{array}{c}
x_{2} \\
x_{2}^{\prime}
\end{array}\right|=M_{2}\left|\begin{array}{c}
x_{20} \\
x_{20}^{\prime}
\end{array}\right|
$$

sont données dans l'appendice.

3. Mouvement dans le champ de fuite. - Les coordonnées de la trajectoire lors de la traversée du champ de fuite ont été déterminées par la même méthode que celle utilisée ci-dessus. Ces coordonnées sont :

$$
\begin{aligned}
x_{1}= & u \int_{0}^{x_{3}} v e B_{2}\left(0,0, x_{3}\right) \mathrm{d} x_{3} \\
& \quad-v \int_{0}^{x_{3}} u \cdot e \cdot B_{2}\left(0,0, x_{3}\right) \mathrm{d} x_{3}+x_{10} u+p x_{10}^{\prime} v \\
x_{2}= & x_{20} w+p_{0} x_{20}^{\prime} t .
\end{aligned}
$$

Les intégrales et les fonctions $u$ et $v$ figurant dans (6) ont été calculées à partir de mesures effectuées sur un modèle.

a) Cas D'Émergence normale. - Les coordonnées deviennent alors :

$$
\begin{aligned}
& x_{1}=x_{10}+x_{10}^{\prime} x_{3}+d_{0} \frac{d_{0}}{r_{0}} \int_{0}^{x_{3} / d}\left[\int_{0}^{x_{3} / d} f(s) \mathrm{d} s\right] \mathrm{d} w \\
& x_{2}=x_{20}+x_{20}^{\prime} x_{3}
\end{aligned}
$$

où $f(s)$ représente l'évolution du champ de fuite. Le champ de fuite provoque donc une déflexion supplémentaire de tout le faisceau s'étendant sur une distance $0,8 d_{0}$ (fig. 3 ) où $d_{0}$ est l'entrefer sur la trajectoire $r=r_{0}$.

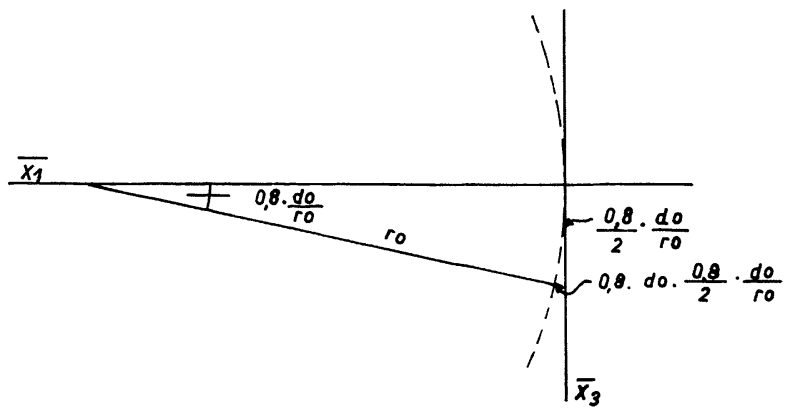

FIG. 3. $-d_{0}=$ écartement des pièces polaires sur la trajectoire centrale $\left(\rho=r_{0}\right)$. 
b) Cas d'émergence non normale. - Si le faisceau quitte l'aimant en formant un angle $\varepsilon$ avec la normale aux pièces polaires, la variation du champ est, sur la trajectoire centrale :

$$
\left(\frac{\partial B_{2}}{\partial x_{1}}\right)_{0,0, x_{3}}=\frac{B_{0} \sin \varepsilon}{d_{0}} f^{\prime}\left(\frac{x_{3} \cos \varepsilon}{d_{0}}\right) .
$$

Par approximations successives, on trouve alors pour les coordonnées de la trajectoire :

$$
\begin{aligned}
x_{1}= & x_{10}-\frac{x_{10}}{r_{0}} \cdot \operatorname{tg} \varepsilon \cdot x_{3} \\
& +\frac{d_{0}}{\cos \varepsilon} \cdot \frac{d_{0}}{r_{0} \cos \varepsilon} \int_{0}^{x_{3} \cos \varepsilon / d_{0}}\left[\int_{0}^{u} f(s) \mathrm{d} s\right] \mathrm{d} u+x_{10}^{\prime} \cdot x_{3}
\end{aligned}
$$

$x_{2}=x_{20}+\frac{x_{20}}{r_{0}} \cdot \operatorname{tg} \varepsilon \cdot x_{3}+x_{20}^{\prime} \cdot x_{3}$.

En comparant (7) et (8), on remarque que l'effet de focalisation dû au champ de fuite se traduit respectivement pour les première et seconde sections principales, par l'apparition des matrices :

$$
\left|\begin{array}{ccc}
1 & 0 & 0 \\
-\frac{\operatorname{tg} \varepsilon}{r_{0}} & 1 & 0 \\
0 & 0 & 1
\end{array}\right| \text { et }\left|\begin{array}{cc}
1 & 0 \\
\frac{\operatorname{tg} \varepsilon}{r_{0}} & 1
\end{array}\right|
$$

4. Conclusion. - a) Les trajectoires et la dispersion sont définies pour un aimant formé d'un secteur à champ non uniforme suivi d'un secteur à champ uniforme par des expressions de la forme (pour chaque section principale) :

$$
\left|\begin{array}{c}
x \\
x^{\prime} \\
\frac{\mathrm{d} p}{p_{0}}
\end{array}\right|_{\text {sortie }}=\left|M_{\mathrm{f}}\right|\left|M_{\mathrm{h}}\right|\left|M_{\mathrm{inh}}\right|\left|M_{\mathrm{f}}\right|\left|\begin{array}{c}
x \\
x^{\prime} \\
\frac{\mathrm{d} p}{p_{0}}
\end{array}\right|_{\text {entrée }}
$$

où $M_{\mathrm{f}}=$ matrice du champ de fuite,

$M_{\mathrm{h}}=$ matrice du champ uniforme,

$M_{\mathrm{inh}}=$ matrice du champ non uniforme.

Les valeurs des éléments sont données en appendice, pour $M_{\mathrm{h}}$ et $M_{\mathrm{inh}}$.

b) Si la source se trouve à la distance $E$ du prisme, la distance de l'image depuis la sortie du prisme est, dans le plan horizontal :

$$
S=-\frac{a_{12}+E a_{11}}{a_{22}+E a_{21}}
$$

et l'agrandissement vaut :

$$
A=a_{11}+E a_{21} .
$$

c) Les électrons d'impulsion $p+\mathrm{d} p$ forment une image déplacée dans la direction $x_{1}$ de :

$$
D=a_{13}+E a_{23} \text { (dispersion) }
$$

et la résolution est donnée par :

$$
R=\frac{L+F}{D}
$$

où $L$ et $P$ sont respectivement les largeurs de l'image et de la fente du détecteur.

\section{DEUXIÈME PARTIE}

\section{Application}

Les calculs précédents ont été appliqués à un modèle composé de deux secteurs, l'un à champ non uniforme de gradient $a=1$, suivi d'un secteur à champ uniforme. Le champ non uniforme assure la focalisation axiale $\left(x_{2}\right)$ et le champ homogène la focalisation radiale $\left(x_{1}\right)$.

La position des images pour chacune des directions principales est déterminée par le choix des angles des secteurs $\Phi$ et $\Psi^{\prime}$ ( $f i g .1$ ), de l'angle d'émergence $\varepsilon_{\mathrm{s}}$ et de la distance de la source au spectromètre $\left(L_{\mathrm{s}}\right)$. La position de l'image, pour des valeurs déterminées des angles, varie entre deux extrêmes, correspondant aux cas où la source se trouve à l'infini et à l'entrée du spectromètre $\left(L_{\mathrm{s}}=0\right)$. Les abaques reprises aux figures 4 à 6 montrent l'évolution de la position, d'une part, de l'image horizontale :

$$
\frac{L_{\mathrm{i}}}{r_{\mathbf{0}}} \quad \text { pour } \quad L_{\mathrm{s}}=0
$$

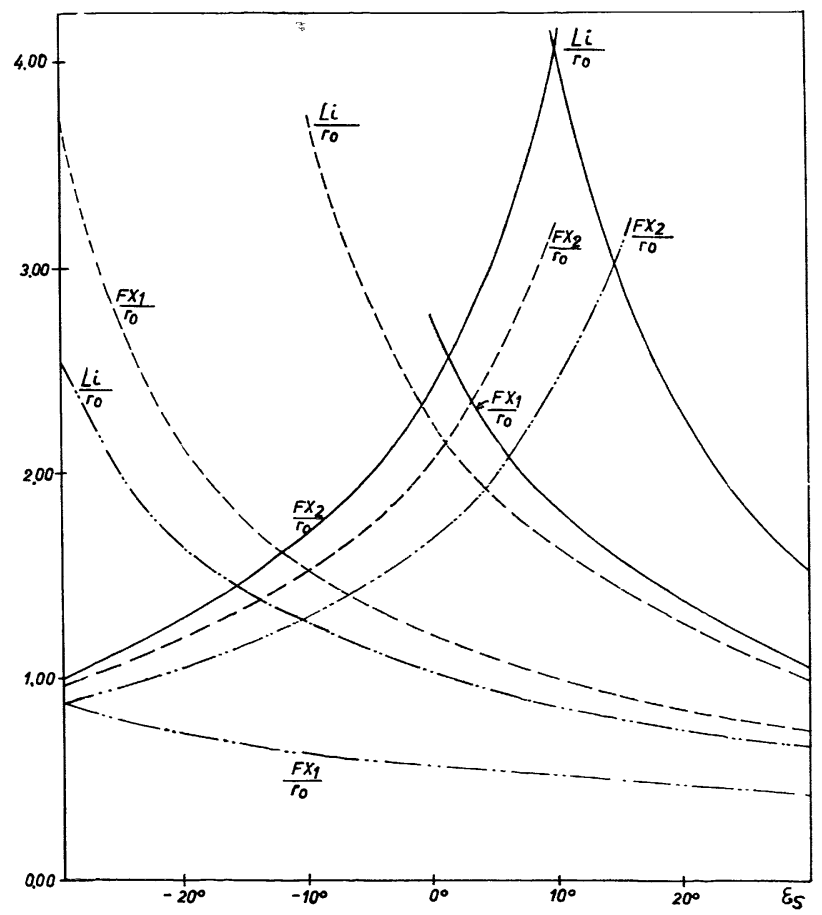

FIG. 4.

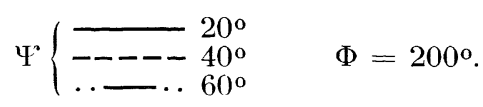


et : $\quad \frac{F x_{1}}{r_{0}}$ pour $L_{\mathrm{s}}=\infty$

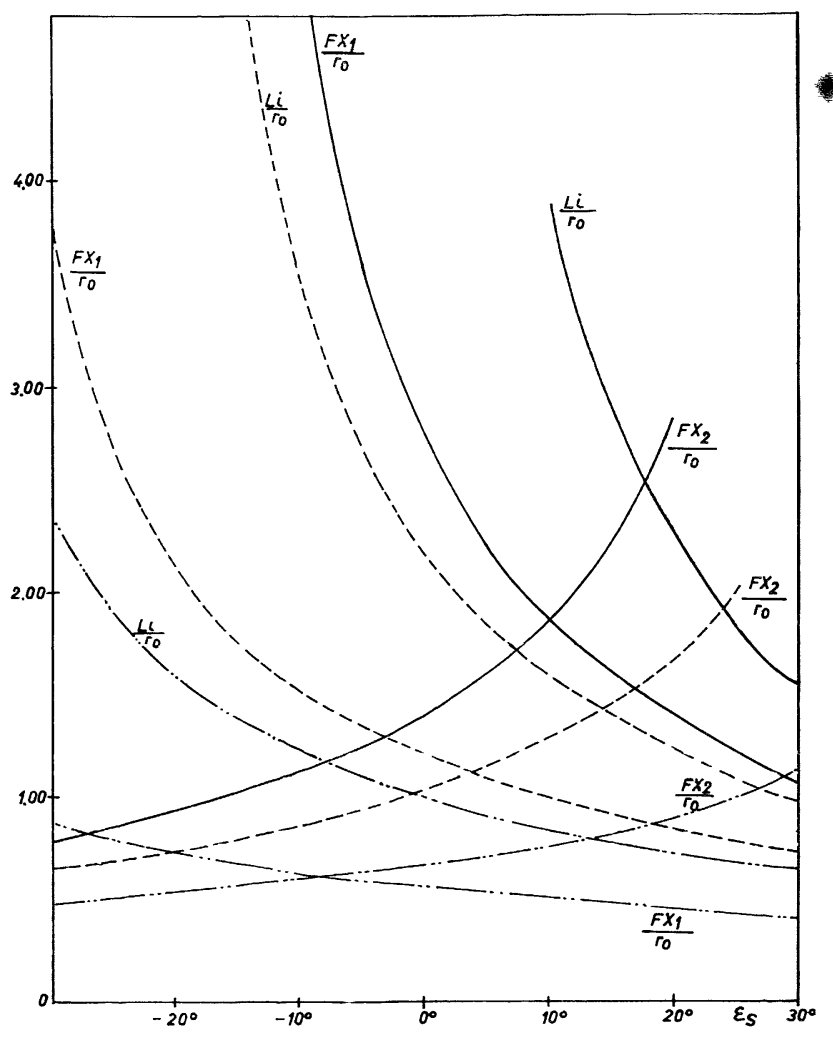

FIG. 5.

$\Psi \cdot\left\{\begin{array}{l}-20^{\circ} \\ -----40^{\circ} \\ \ldots-\ldots 60^{\circ}\end{array} \quad \Phi=210^{\circ}\right.$ et, d'autre part, de l'image verticale :

$$
\frac{F x_{2}}{r_{0}} \text { pour } L_{\mathrm{s}}=\infty .
$$

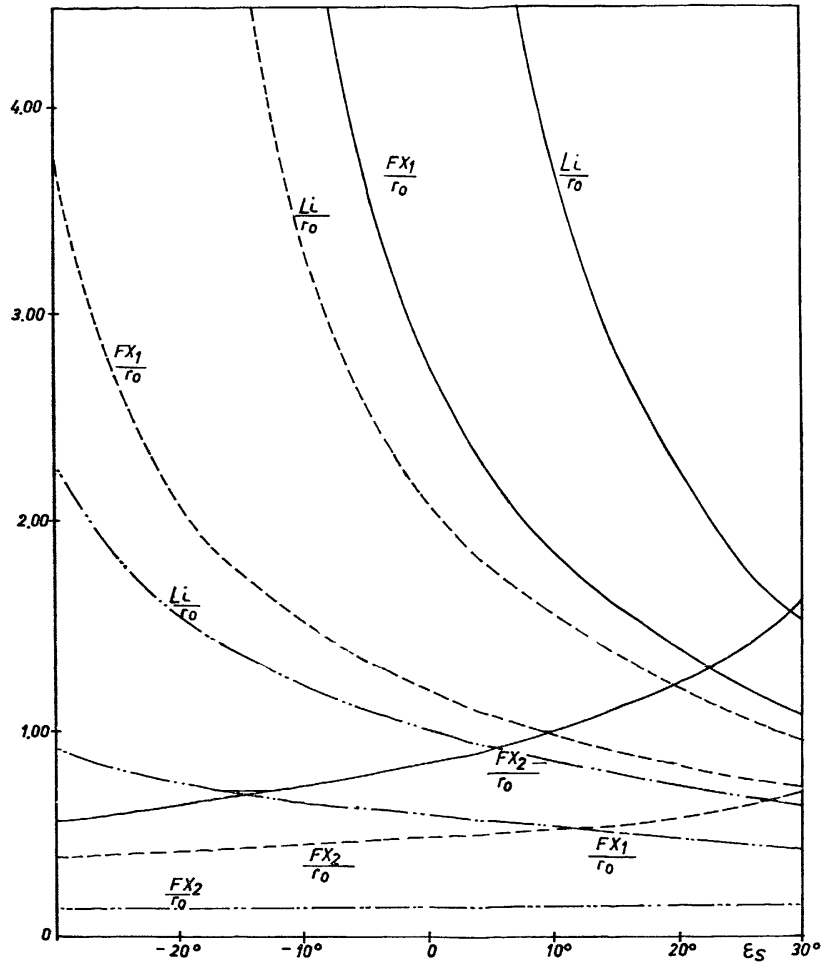

FIG. 6 .

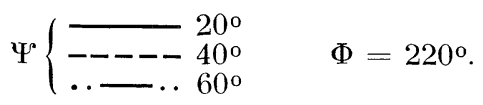

\section{APPENDICE}

Eléments de matrice des secteurs

Première section principale $\left(x_{2}=0\right)$ :

$\begin{array}{ccc}\text { ÉLÉments } & a \leqslant 1 & a>1 \\ - & \cos (1-a)^{1 / 2} \varphi & \cosh (a-1)^{1 / 2} \varphi \\ a_{11} & \frac{r_{0}}{(1-a)^{1 / 2}} \sin (1-a)^{1 / 2} \varphi & \frac{r_{0}}{(a-1)^{1 / 2}} \sinh (1-a)^{1 / 2} \varphi \\ a_{12} & \frac{r_{0}}{1-a}\left[\cos (1-a)^{1 / 2} \varphi-1\right] & \frac{r_{0}}{a-1}\left[\cosh (1-a)^{1 / 2} \varphi-1\right] \\ a_{13} & -\frac{(1-a)^{1 / 2}}{r_{0}} \sin (1-a)^{1 / 2} \varphi & -\frac{(a-1)^{1 / 2}}{r_{0}} \sinh (a-1)^{1 / 2} \varphi \\ a_{21} & \cos (1-a)^{1 / 2} \varphi & \cosh (a-1)^{1 / 2} \varphi \\ a_{22} & -\frac{\sin (1-a)^{1 / 2} \varphi}{(1-a)^{1 / 2}} & -\frac{\sinh (a-1)^{1 / 2} \varphi}{(a-1)^{1 / 2}} \\ a_{23} & 0 & 0 \\ a_{31}=a_{32} & 1 & 1 \\ a_{33} & & \end{array}$


Deuxième section principale $\left(x_{1}=0\right)$ :

\begin{tabular}{cccc} 
ÉLÉMENTS & $a>0$ & $a<0$ & $a=0$ \\
\hline- & - & - & - \\
$b_{11}$ & $\cos \sqrt{a} \varphi$ & $\cosh \sqrt{a} \cdot \varphi$ & 1 \\
$b_{12}$ & $\frac{r_{0}}{\sqrt{a}} \sin \sqrt{a} \cdot \varphi$ & $\frac{r_{0}}{\sqrt{a}} \sinh \sqrt{a} \cdot \varphi$ & $r_{0} \varphi$ \\
$b_{21}$ & $-\frac{\sqrt{a}}{r_{0}} \sin \sqrt{a} \cdot \varphi$ & $-\frac{\sqrt{a}}{r_{0}} \sinh \sqrt{a} \cdot \varphi$ & 0 \\
$b_{22}$ & $\cos \sqrt{a} \cdot \varphi$ & $\cosh \sqrt{a} \cdot \varphi$ & 1
\end{tabular}

[1] Von Egrdy (T.), Thèse, T.H., Munich, 1961.

[2] Von Egidy (T.), Ann. Physik, 7, Bd. 9, Heft 5-6, 1962.

[3] CotTe (M.), Ann. Physique, 1938, 10, 333.

[4] CotTe (M.), C. R. Acad. Sci. Pavis, 1949, 228, 337. 